1999;45:154-161), Clark GD and Naebels JL of Baylor College of Medicine, Houston, point out the importance of genotype-phenotype correlations in understanding the overlapping of clinical/pathological manifestations of migration disorders and the value in genetic counseling. Asymptomatic mothers of children with $D C X$ mutations are at risk of further transmitting the disorder, whereas no germline transmission of LIS1 mutations have been described.

\title{
NODULAR NEURONAL HETEROTOPIA
}

Brain tissue from 4 children with intractable epilepsy and subcortical or periventricular nodular heterotopia of different etiologies (megalencephaly, cortical dysplasia, polymicrogyria) was examined at the University of Oxford, Radcliffe Infirmary, UK. Histological and carbocyanine dye (DiI) tracing techniques showed fibers surrounding nodules and connectivity between adjacent nodules. Immunohistochemical tests for calretinin and neuropeptide $Y$ (NPY), normally expressed in GABAergic cortical interneurons, found numerous calretinin-positive neurons (CPN) within nodules with incomplete differentiation, abnormal clusters of CPN in the overlying cortical plate, and many cell processes positive for NPY. Heterotopic nodules were associated with malformation in the overlying cortex and had limited connectivity with other brain regions. Abnormal connectivity could affect the balance of excitation and inhibition in neuronal circuits, leading to epileptogenic activity. (Hannan AJ, Servotte S, Katsnelson A et al. Characterization of nodular neuronal heterotopia in children. Brain Feb 1999;122:219-238). (Dr Zoltan Molnar, Institut de Biologie Cellulaire et de Morphologie, Rue du Bugnon 9, 1005 Lausanne, Switzerland).

COMMENT. The abnormal structure, composition, and connections between nodules and the overlying cortex may explain the epileptogenicity of neuronal heterotopias and its propagation to other brain regions.

\section{POSTERIOR FOSSA MALFORMATIONS AND EPILEPSY}

Risk factors for epilepsy in children with posterior fossa malformations (PFM) were studied in 22 cases of PFM with epilepsy (41\%), and 32 without epilepsy (59\%), seen at the University of Bologna, Italy. The most common PFMs were cerebellar hypoplasia (43\%), Dandy-Walker (D-W) complex (37\%), and Arnold-Chiari (A-C) malformation (13\%). Epilepsy recurred most frequently in AC malformation (71\%), compared to $35 \%$ in D-W complex, and $35 \%$ in cerebellar hypoplasia. Risk factors for epilepsy in PFM cases were cerebellar lesions plus familial antecendents for epilepsy and/or febrile convulsions; 50\% in PFM cases with epilepsy, and $9 \%$ in PFM without epilepsy. Epilepsy was mainly partial in $77 \%$; benign epilepsies and febrile convulsions occurred in 27\%. (Parmeggiani A, Posar A, Scaduto MC et al. Posterior fossa malformations and epilepsy. I Child Neurol Feb 1999;14:113-117). (Respond: Dr Antonia Parmeggiani, Department of Child Neurology and Psychiatry, Neurological Institute, University of Bologna, via Ugo Foscolo 7 , 40123 Bologna, Italy).

COMMENT. Epilepsy may occur in $40 \%$ of children with posterior fossa malformation. A family history of epilepsy and/or febrile convulsions are significant risk factors for epilepsy in children with posterior fossa malformation.

Posterior fossa pathology other than malformation can predispose to epilepsy. Among 291 children treated for intracranial tumor at the Mayo Clinic over a 10 year period, seizures occurred in $17 \%$ - in $25 \%$ of patients with supratentorial tumors and in $12 \%$ of those with infratentorial tumors. The 